\title{
Lepton-flavor-violating $\tau$ decay prospects at SuperKEKB/Belle II
}

\author{
Kenji Inami ${ }^{* \dagger}$ \\ Nagoya Univ. \\ E-mail: kenji@hepl.phys.nagoya-u.ac.jp
}

Lepton flavor violating $\tau$ decay is a clear signature of new physics beyond the Standard Model. The Belle II experiment aims to record $50 \mathrm{ab}^{-1}$ of data at the SuperKEKB energy-asymmetric $e^{+} e^{-}$collider, which is 50 times larger amount compared to the current experiments. The anticipated high statistics data sample has excellent sensitivity to lepton flavor violating $\tau$ lepton decays. The expected sensitivity of the branching ratio is $\mathrm{O}\left(10^{-9}\right) \sim \mathrm{O}\left(10^{-10}\right)$, which depends on the background situation.

38th International Conference on High Energy Physics

3-10 August 2016

Chicago, USA

* Speaker.

${ }^{\dagger}$ Belle/Belle II collaboration 


\section{Lepton flavor violation in $\tau$ decay}

Lepton flavor violation (LFV) in $\tau$ decay is one of main topics at the B-factory experiment, as a probe of new physics (NP) beyond the standard model (SM). In the SM, LFV is highly suppressed [1], which is impossible to access by actual experiments. However, many extensions of the SM predict LFV decays. Their BRs are enhanced as high as current experimental sensitivity. Therefore, observation of LFV decays is a clear signature of NP. Tau lepton is a heaviest charged lepton, so it opens many possible LFV decay modes, which classifies the NP models.

\section{Current status}

The current best sensitivity is obtained by the previous B-factory experiments. We have performed the LFV $\tau$ decay searches using huge amount of data obtained by the KEKB/Belle experiment $[2,3]$. KEKB is asymmetric energy $e^{+} e^{-}$collider with $\sqrt{s}=10.58 \mathrm{GeV}$ and realized world highest luminosity. The cross section of $\tau$ pair production is similar with the one of $b$ quark pair production. So, a B-factory is also a $\tau$-factory. Belle detector has collected the data with good tracking reconstruction and particle identification (ID) system. The lepton ID is important for the LFV searches. Belle has achieved $90 \%$ efficiency with the fake rate of $\mathrm{O}(0.1) \%$ for electrons and $\mathrm{O}(1) \%$ for muons. Finally, we have collected $\sim 10^{9} \tau$ pairs.

In the analysis, we have selected $\tau$ pair events, in which one $\tau$ decays to one charged particle and neutrals, and another decays to LFV signal mode that is fully reconstructed. The number of signal events is basically extracted using the invariant mass $m$ of the signal side and $\Delta E$ that is residual of reconstructed energy minus beam energy. From the data distribution on $m-\Delta E$ plot shown in Fig. 1(left), we estimate the number of background (BG) events in the signal region using sideband data and Monte-Carlo (MC) expectation.

The search for $\tau \rightarrow 3$ leptons is performed using the Belle data of $\sim 7 \times 10^{8} \tau$ pairs. We found no event in the signal region and set the upper limit (UL) of BR to be $(1.5 \sim 2.7) \times 10^{-8}$ at $90 \%$ confidence level [4]. We also found almost BG free condition from the sideband data, because of good lepton ID. The search for $\tau t o \mu \gamma$ and $e \gamma$ is performed based on $\sim 4.8 \times 10^{8} \tau$ pairs and results in obtaining several events in the signal region, however no meaningful excess from the sideband distribution, as shown in Fig. 1(right). The obtained UL is $4.5 \times 10^{-8}$ for $\tau \rightarrow \mu \gamma$ and $1.2 \times 10^{-7}$ for $e \gamma$ [5]. The dominant BG is general $\tau$ pair events with hard initial state radiation and leptonic $\tau$ decay. It is hardly irreducible BG.

The current results for many LFV decay modes are summarized in Fig. 2. B-factories, Belle and $\mathrm{BaBar}$ experiments, have improved the sensitivity by about one order of magnitude from the older CLEO experiment. Belle, BaBar and recently LHCb are reaching $\mathrm{O}\left(10^{-8}\right) \mathrm{BR}$ sensitivity. As shown in the results, the decay modes of $\tau \rightarrow$ 3leptons and lepton plus mesons (decaying to charged particles) show better sensitivity because of less BG, compared to $\tau \rightarrow l \gamma$.

\section{Future prospect at Belle II}

We are upgrading the experiment, to achieve further extended sensitivity by collecting more data with better detector performance. The Belle II experiment will accumulate $50 \mathrm{ab}^{-1}$ integrated luminosity, which corresponds to $\sim 5 \times 10^{10} \tau$ pair events, with the upgraded detector [6]. 

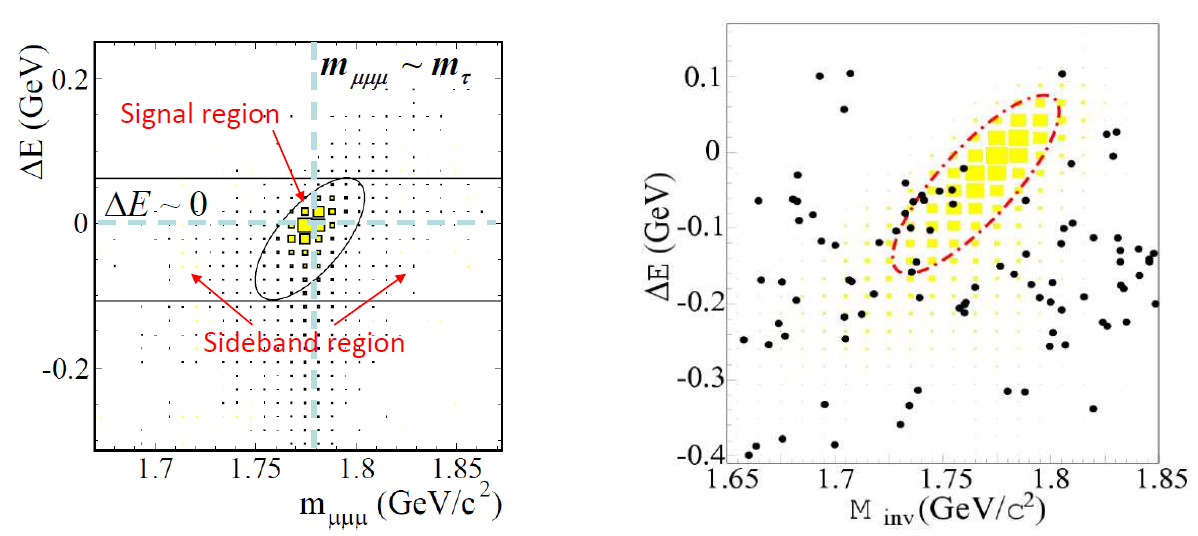

Figure 1: The $m-\Delta E$ distributions for $\tau \rightarrow \mu \mu \mu \mathrm{MC}$ (left) and $\tau \rightarrow \mu \gamma$ data and MC (right). The horizontal axis is the invariant mass $m$ and the vertical is $\Delta E$. Boxes indicate the signal MC expectation and dots are data. The ellipse shows the signal region.

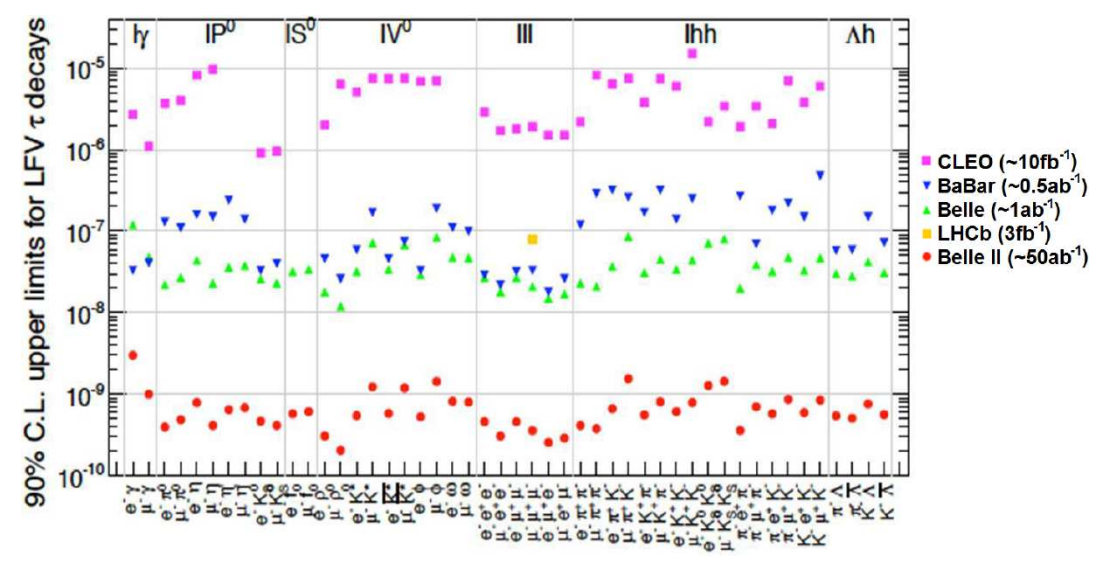

Figure 2: The upper limits of the BRs for LFV $\tau$ decays obtained by CLEO, BaBar, Belle and LHCb experiments and the prospects for the Belle II experiment.

The BR sensitivity depends on the integrated luminosity, $\mathscr{L}$, however, the dependence is different due to the BG condition. For $\tau \rightarrow l \gamma$ mode, the sensitivity is proportional to $1 / \sqrt{\mathscr{L}}$, because of the non-negrigible BG from $e^{+} e^{-} \rightarrow \tau^{+} \tau^{-} \gamma$. On the other hand, the sensitivity of $\tau \rightarrow 3$ leptons and lepton plus mesons depends on $1 / \mathscr{L}$ because of negligible $\mathrm{BG}$ events reduced by particle ID and meson mass restriction. Therefore, it is important to reduce BG (or improve signal-to-noise ratio, $\mathrm{S} / \mathrm{N}$ ), to achieve further sensitivity. The $\mathrm{S} / \mathrm{N}$ for $\tau \rightarrow l \gamma$ will improve, if $\gamma$ energy resolution improves. In the Belle II experiment, less material before electromagnetic calorimeter will improve the resolution, however, we need to care the deterioration due to the high beam BG condition.

The future prospects of the BR sensitivity is shown in Fig. 2 and 3, which are extracted using the BG situation in the latest analysis. The BR of $\tau \rightarrow \mu \mu \mu$ is expected to reach $\mathrm{O}\left(10^{-10}\right)$ at 50 $\mathrm{ab}^{-1}$. On the contrary, the BR of $\tau \rightarrow \mu \gamma$ will be $\sim 2 \times 10^{-9}$, which is limited by non-negligible BG.

The theoretical predictions of the BR of $\tau \rightarrow \mu \gamma$ and $\mu \mu \mu$ are shown in Table 1. Now, the experiments are limiting the parameter space of NP models and reaching the region of large $\tan \beta$ 


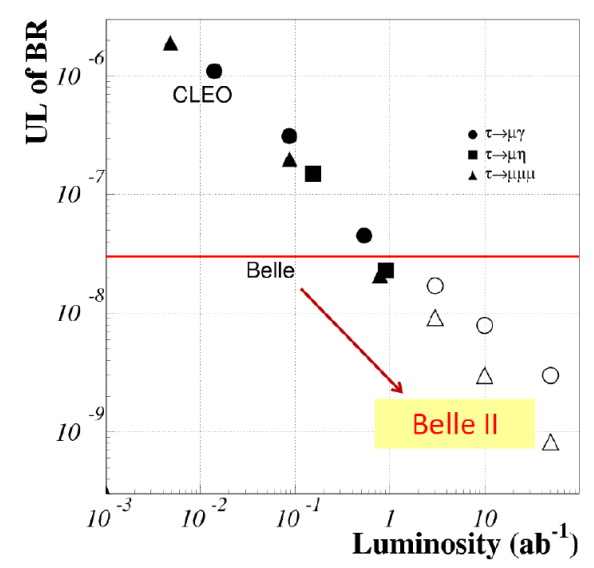

Figure 3: The future prospects on the upper limit of the BR as a function of integrated luminosity. Filled marks show the experimental results obtained by CLEO and Belle experiments, and open marks indicate the prospects at Belle II experiment.

and small SUSY/Higgs mass in some SUSY models. The Belle II experiment will explore larger parameter space and may find NP.

Table 1: Theoretical predictions of the branching ratio for $\tau \rightarrow \mu \gamma$ and $\tau \rightarrow \mu \mu \mu$.

\begin{tabular}{l|cc}
\hline Model & $\tau \rightarrow \mu \gamma$ & $\tau \rightarrow \mu \mu \mu$ \\
\hline SM + heavy $v_{R}[7]$ & $10^{-9}$ & $10^{-10}$ \\
Non-universal $Z^{\prime}[8]$ & $10^{-9}$ & $10^{-8}$ \\
SUSY SO(10) [9] & $10^{-8}$ & $10^{-10}$ \\
mSUGRA + seesaw [10] & $10^{-7}$ & $10^{-9}$ \\
SUSY Higgs [11] & $10^{-10}$ & $10^{-7}$ \\
\hline
\end{tabular}

\section{Summary}

Previous B factory experiments have reached $\mathrm{O}\left(10^{-8}\right)$ BR sensitivity using $\sim 10^{9} \tau$ pair events. Belle II experiment will start soon and collect $\sim 5 \times 10^{10} \tau$ pair events. LFV search sensitivity depends on the statistics, however the slope is different due to the BG condition. The BG-free modes, such as $\tau \rightarrow 3$ leptons, can be reached to $\mathrm{O}\left(10^{-10}\right)$ BR sensitivity, although the mode $\tau \rightarrow l \gamma$ will be $\mathrm{O}\left(10^{-9}\right)$ that highly depends on the number of BG events in the signal region.

\section{References}

[1] T. P. Cheng and L. F. Li, Phys. Rev. D 16, 1425 (1977).

[2] S. Kurokawa and E. Kikutani, Nucl. Instr. Meth. A 499, 1 (2003), and other papers included in this Volume.

[3] A. Abashian et al. [Belle Collaboration], Nucl. Instr. and Meth. A 479, 117 (2002). 
[4] K. Hayasaka et al. [Belle Collaboration], Phys. Lett. B 687, 139 (2010).

[5] K. Hayasaka et al. [Belle Collaboration], Phys. Lett. B 666, 16 (2008).

[6] T. Abe et al. [Belle-II Collaboration], arXiv:1011.0352 [physics.ins-det]; see also relevant presentations at ICHEP2016 conference.

[7] G. Cvetic, C. Dib, C. S. Kim and J. D. Kim, Phys. Rev. D 66, 034008 (2002), [Erratum-ibid. D 68, 059901 (2003)].

[8] C. X. Yue, Y. m. Zhang and L. j. Liu, Phys. Lett. B 547, 252 (2002).

[9] T. Fukuyama, T. Kikuchi and N. Okada, Phys. Rev. D 68, 033012 (2003).

[10] J. R. Ellis, J. Hisano, M. Raidal and Y. Shimizu, Phys. Rev. D 66, 115013 (2002).

[11] A. Brignole and A. Rossi, Phys. Lett. B 566, 217 (2003). 